\title{
Comportement et dimensionnement des ouvrages en Terre Armée sous actions dynamiques
}

\section{A. DHOUIB}

Chef de la Section Études et Calculs automatiques GÉO-SIGMA

170 , rue de la DivisionLeclerc,

91160 Saulx-les-Chartreux

Ingénieur conseil EPLM Expertises 2, rue Gutenberg, 91620 Nozay
Cette présentation a pour but de décrire le comportement avant rupture des massifs en sols renforcés soumis à des actions dynamiques.

Pour cela, on effectue, dans un premier temps; une étude comparative entre des mesures japonaises sur modèles en semi-grandeur et une modélisation par éléments finis basée sur une approche linéaire équivalente.

On établit, dans un second volet, une synthèse des différentes méthodes de dimensionnement dans ce domaine. Des critiques et des réflexions sur le dimensionnement des ouvrages en Terre Armée soumis à des actions dynamiques achèvent la présente communication.

\section{Analysis and design of reinforced earth structures under dynamic efforts}

\footnotetext{
느 The behaviour of reinforced soils under dynamic actions before failure is analysed.

First, a comparative study between Japanese semi-full scale experiments and finite elements method is carried out. assuming an equivalent linear response of the soil.

Then, a synthesis of recent design methods of reinforced earth structures is submitted.

Critical analysis and comments on current design techniques in the event of dynamic forces are proposed.
} 


\section{NOTATIONS} inclusion.

$\Delta \mathrm{T}_{\mathrm{d}} \quad$ : incrément de traction dû à l'action dynamique.

$\mathrm{T} \quad$ : traction totale $\left(=\mathrm{T}_{\mathrm{i}}+\Delta \mathrm{T}_{\mathrm{is}}\right)$.

$\mathrm{T}_{\max }$ : traction totale maximale.

D : largeur de la zone instable.

$\Delta \mathrm{D}$ : élargissement de la zone instable sous l'ef fet de l'action dynamique.

$D^{\prime}$ : largeur totale $(=D+\Delta D)$.

$\mathrm{H}$ : hauteur du massif renforcé.

L. : longueur de l'inclusion.

x : abscisse suivant L

A : pourcentage d'amortissement.

$\left[\mathrm{K}^{*}\right]$ : matrice de rigidité globale complexe,

$\mathrm{R}_{\mathrm{n}} \quad$ : résistancé normale de l'inclusion.

$R_{c}$ : résistance statique au frottement sol inclusion.

$R_{n}^{\prime} \quad$ : résistance dynamique au frottement sol inclusion.

$\mathrm{m}_{\mathrm{h}}$ : rapport de l'accélération horizontale $\mathrm{a}_{\mathrm{h}}$ à la

$P_{4} \quad$ : poussée du remblai derrière le massif armé, due à l'action dynamique.

$F_{1} \quad$ : force d'inertie.
$\mathrm{T}_{\mathrm{s}} \quad$ : traction statique développée le long d'une gravité g.

On mettra particulièrement l'accent sur le calcul et le dimensionnement des ouvrages en Terre Armée sous actions dynamiques,

\section{2}

\section{Comportement avant rupture des sols renforcés}

\section{1}

\section{Paramètres d'étude et observations expérimentales}

On envisage de suivre dans la présente étude : - l'évolution des tractions statiques et dynamiques le long de l'inclusion:

- la distribution et le lieu des tractions totales maximales.

Nous rappelons que, dès que le sol subit des déplacements, il se développe une traction le long de l'ínclusion. Son évolution est généralement analogue à celle de la figure 1.

Lorsque le massif renforcé est soumis à une action dynamique, les expérimentations de Richardson [9] et Chida $[3,4]$ mettent en évidence les points suivants : - Un incrément de traction dynamique $\Delta \mathrm{T}_{\mathrm{s}}$ se développe. Il croit avec l'accélération (on désignera par m, le rapport de l'accélération horizontale $a_{n}$ à la gravité g). Ainsi, la traction totale à prendre en compte dans le calcul dynamique résulte d'une partie statique $(\mathrm{T}$ ) et d'une partie dynamique $\left(\Delta \mathrm{t}_{\mathrm{d}}\right)$, soit :

$$
\mathrm{T}=\mathrm{T}_{s}+\Delta \mathrm{T}_{\mathrm{s}}
$$

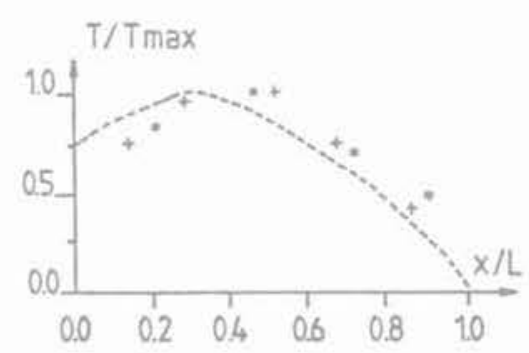

$+\quad$ Mesures sur ouvrages réels

(mur d'Incarville, F. Schlosser, blpc 63, 1973)

Calcul E.F. Shafiee

CERMES, 1986.

Mesures sur modèles réduits tridimensionnels, Dhouib et al. CERMES, 1984

En France, seuls quelques articles émanant de Bastick [1,2]. Schlosser et al. [11] ont été publiés sur le sujet malgré de nombreux essais et calculs effectués, par la société Terre Armée. On cite également la thèse de Madani (1979) qui aborde «l'étude du mécanisme interne et du comportement dynamique de la terre armée à l'appareil triaxial n) et le travail de fin d'étude de Khouaja qui constitue un bon support bibliographique du $\alpha$ Dimensionnement des ouvrages en terre armée aux sollicitations sismiques » (1981).

Le but de cette présentation est de décrire le comportement avant rupture des ouvrages armés, et de faire le point sur l'état actuel de connaissance dans le domaine de la dynamique des sols renforcés.
FiG.1 Evolution de la traction statique dans une inclusion Terre Armée. Evolution of static tensile force in reinforced earth strip.

- L'action dynamique modifie, si l'on considère les courbes enveloppes des tractions maximales $T_{\text {my }}$ l'évolution de celles-ci, en fonction de la profondeur. On remarque que $T_{\max }$ croit lorsque $m_{h}$ augmente.

- L'examen des distributions de $\mathrm{T}_{\operatorname{mix}}$ montre que l'excitation dynamique fait croître, en considérant également 
des courbes enveloppes, la largeur de la zone instable. Cet élargissement $(\Delta \mathrm{D})$ croît avec l'accélération $\mathrm{m}_{\mathrm{h}}$.

Ainsi, la largeur de la zone « active» est:

$D^{\prime}=D+\Delta D$ (D étant sa largeur sous charge statique).

\section{2}

\section{Modélisation numérique proposée}

On envisage, dans le cadre de cette modélisation, de suivre le comportement des ouvrages en Terre Armée à travers quelques mesures sur modèle en semi-grandeur expérimenté par Chida [3], et des calculs par éléments finis effectués par Dhouib et al. [5 à 7].

Les résultats numériques sont obtenus à partir đ'une analyse linéaire équivalente. La procédure de linéarisation repose sur la recherche d'un pourcentage d'amortissement A et d'un module de cisaillement G', tous deux équivalents. La loi de comportement s'écrit sous une forme analogue à la loi de Hooke généralisée et on résout dans un champ complexe, le système suivant:

avec :

$$
\left[\mathrm{K}^{*}\right] .\{\mathrm{U}(\mathrm{t})\}=\{\mathrm{F}(\mathrm{t})\}
$$

$$
\left[K^{*}\right]=[K]\left(1-2 A^{2}+2 A i \sqrt{1-A^{2}}\right)
$$

[K] étant la matrice de rigidité globale :

$\{\mathrm{U}(\mathrm{t})\}$ et $(\mathrm{F}(\mathrm{t}))$ désignent respectivement les vecteurs des déplacements (les inconnus du problème) et des forces nodales (était initial statique et excitation dynamique).

Cette modélisation issue d'une linéarisation de Lysmer (1975) a le mérite d'être simple et ne nécessite que la mesure des deux paramètres d'élasticité (le module de cisaillement $\mathrm{G}$ et le coefficient de Poisson v) et du coefficient de perte $2 \mathrm{~A}$.

\section{3}

\section{Résultats comparatifs et analyse du comportement}

Les calculs par éléments finis effectués dans le cadre du modèle linéaire équivalent pour divers niveaux d'accélération $m_{n}\left(=a_{n} / g\right)$ sont comparés aux résultats expérimentaux obtenus par Chida et al, sur un mur en Terre Armée en semi-grandeur $[3,4]$.

\subsection{1}

\section{Evolution des tractions statiques et dynamiques dans l'inclusion}

Sur la figure 2, on donne l'évolution de la traction totale (statique + dynamique) le long de l'inclusion $n^{\circ} 7$ du modèle japonais de Chida et pour plusieurs niveaux d'accélération horizontale $a_{\mathrm{t}}$. La figure 2.a illustre les mesures expérimentales pour $m_{n}=0,1$ et $m_{n}=0,4$ alors que sur la figure 2.b sont présentées les valeurs théoriques pour $m_{n}=0,1-0,25$ et 0,40 à une fréquence de $2 \mathrm{~Hz}$.

A la lumière de ces résultats théoriques et expérimentaux, il y a lieu de constater que :

- l'incrément de traction dynamique $\Delta \mathrm{T}_{d}$ développé dans l'inclusion augmente avec le rapport $\mathrm{m}_{n}$ croissant;

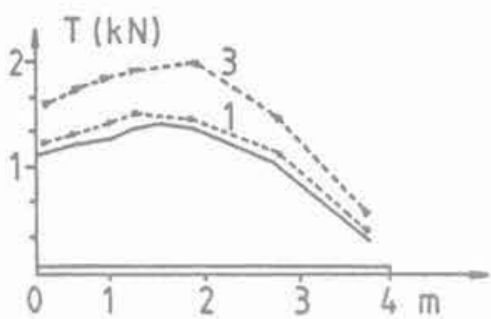

a. Expérience, Chida [4]

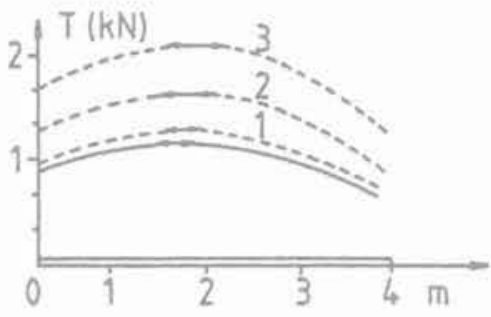

b. Calcul E.F., Dhouib [7]

b. Finite elements analysis

ææ Statique

-.... Totales (statiques + dynamiques)

$1: m_{h}=a_{h} / g=0,10$

$2: m_{n} \quad=0,25$

$3: m_{n}^{n} \quad=0,40$

FG. 2 Évolution des tractions totales $\mathrm{T}$ dans l'armature $n^{\circ} 7$.

Evolution of the whole tensile force $T$ in the strip $n^{6} 7$.

- la traction totale $\left(\mathrm{T}=\mathrm{T}_{4}+\Delta \mathrm{T}_{\mathrm{f}}\right)$ présente un maximum situé au-delà de son homologue statique. Ce maximum s'éloigne de ce dernier lorsque $m_{\mathrm{n}}$ augmente :

- l'approche linéaire équivalente est incapable de tenir compte des non-linéarités pour des accélérations élevées $\left(m_{h}=0,25\right.$ et $\left.m_{h}=0,40\right)$. Cependant, elle constitue un « compromis » et permet de mieux appréhender par le processus itératif et le caractère dissipatif représenté à l'aide du module (complexe) équivalent, les déformations d'origine non linéaires pour des faibles accélérations $\left(m_{h}=0,10\right.$ à 0,15$)$.

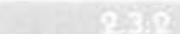

\section{Distribution des tractions maximales}

Les courbes de variations théoriques (calcul E.F.) des tractions totales maximales en fonction de la profondeur (cf. Fig. 3 et 4) montrent que :

- en l'absence de la surcharge (trapézoïdale), l'évolution des tractions statiques maximales fluctue entre les lignes « Ko » et « Ka », résultats qui concordent avec les observations expérimentales sur modèles réduits (Richardson, 1974) et sur ouvrages réels (Chida, 1980). Les actions dynamiques font croître les distributions des tractions maximales;

- la surcharge a pour effet d'éloigner les distributions des tractions maximales statiques et dynamiques des lignes $\mathrm{K}_{0}$ et $\mathrm{K}_{\mathrm{a}}$ (Fig. 4).

On note bien, dans les deux cas, que les valeurs de $\mathrm{T}_{\max }$ augmentent lorsque $\mathrm{m}_{4}$ croît. 


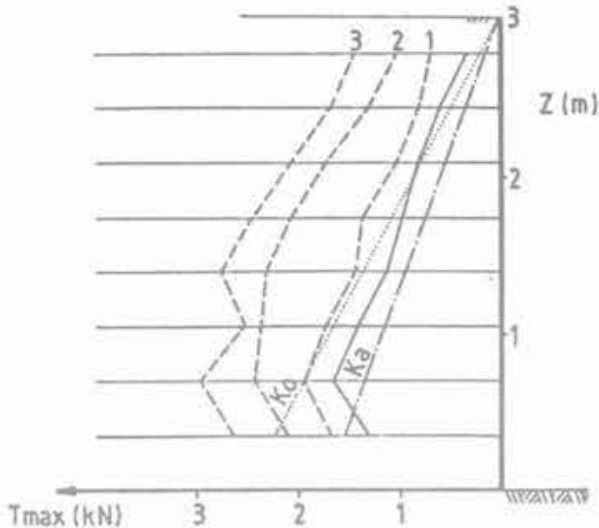

F16. 3 Distribution des tractions totales maximales $\mathrm{T}_{\max }$ (calcul E.F.).

Distribution of theorical maximum tensile force $T_{\max }$ (F.E. analysis).

Statique

Totales (statiques + dynamiques)

$1: m_{b}=a_{1} / g=0,10$

$2: m_{n} \quad=0,25$

$3: \mathrm{m}_{\mathrm{h}} \quad=0,40$

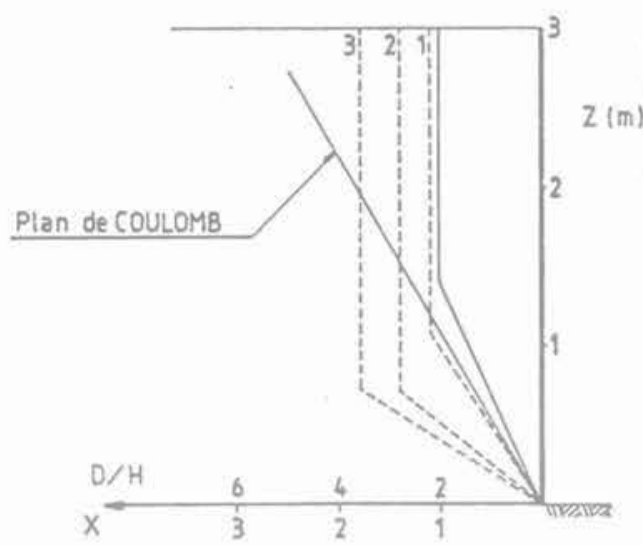

FG.5 Lieu des tractions maximales $\mathrm{T}_{\text {max }}$ (calcul E.F.).

Locus of maximum tensile force $T_{\text {mix }}$

(F.E. analysis).

- Statique

Totale (statiques + dynamiques)

$1: m_{h}=a_{h} / g=0,10$

$2: m_{n} \quad=0,25$

$3: m_{h} \quad=0,40$

\subsection{3}

\section{Lieu des tractions maximales}

Le lieu des tractions maximales représente l'ensemble des points oủ les tractions sont maximales. $\mathrm{Ce}$ qui permet de localiser la surface de rupture potentielle et de définir la largeur de la zone instable.

L'examen des figures 5 et 6 montre, en considérant des ( courbes enveloppes $),$ que :

- l'action dynamique élargit la zone instable d'un incrément de largeur $\Delta \mathrm{D}$;

- l'incrément $\Delta \mathrm{D}$ augmente avec le rapport $\mathrm{m}_{\mathrm{h}}$ croissant;

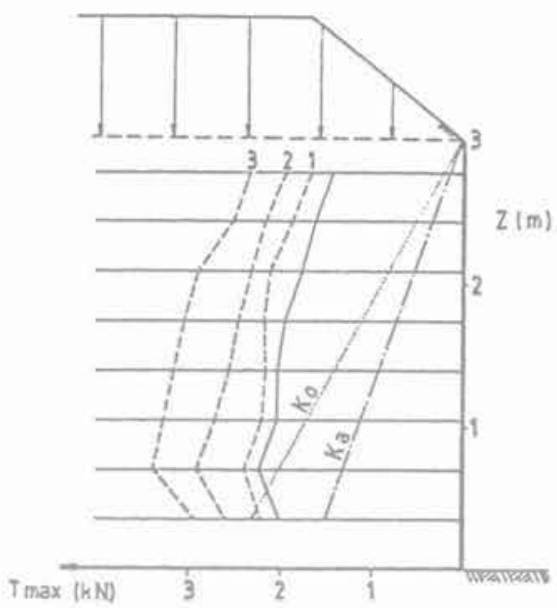

FG. 4 Distribution des tractions totales maximales $\mathrm{T}_{\max }$ : incidence de la surcharge (calcul E.F.).

Distribution of maximum tensile force $T_{\operatorname{mix}}$ Overloading effect (F.E. analysis).

Statique

Totales (statiques + dynamiques)

$1: m_{b}=a_{n} / g=0,10$

$2: m \quad=0,25$

$3: m_{b}$

$=0,40$

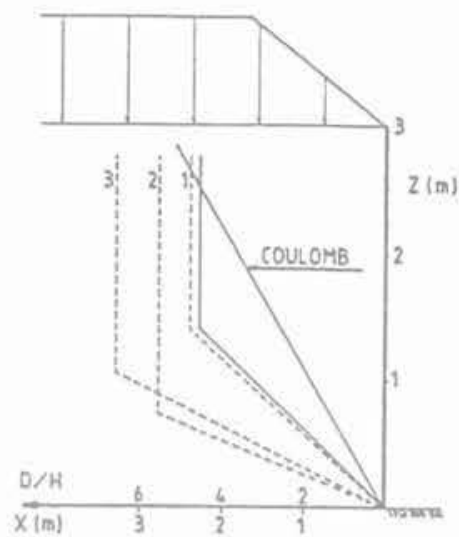

FIG. b Lieu des tractions maximales T effet de la surcharge (calcul E.F.) Locus of maximum tensile force $T$. Overloading effect (F.E. analysis).

Statique
Totales (statiques + dynamiques)
$\begin{array}{ll}1: m_{h}=a_{h} / g & =0,10 \\ 2: m_{h} & =0,25 \\ 3: m_{h} & =0,40\end{array}$

- la surcharge a pour effet de croître l'étendue de la zone instable.

\subsection{6 \\ Évolution des déplacements et phénomènes d'amplification}

L'évolution des déplacements à travers diverses séquences de la sollicitation dynamique (I'état initial étant la dernière phase de construction du mur) conduit à énumérer les points suivants :

- l'action dynamique modifie le mode de déplacement de l'ouvrage renforcé. Les mouvements les plus impor- 
tants sont obtenus, dans le cas d'un mur en Terre Armée, dans la partie supérieure du mur;

- les déplacements d'origine dynamique augmentent avec le rapport $m_{n}$ croissant;

- les accélérations horizontales maximales varient peu en fonction de la profondeur. Leur variation reste à peu près constante dans le tiers inférieur du massif pour des faibles valeurs de $m_{h}$. Lorsque $m_{n}$ croit, un phénomène d'amplification apparait dans le reste de l'ouvrage armé, se traduisant ainsi par une augmentation des accélérations maximales. Ce dernjer résultat semble être en bon accord qualitatif avec les calculs théoriques effectués par le programme de calcul sismique SHAKE de Seed et Idriss [9] et par le code d'éléments finis LEVSFC développé par Idriss à Berkeley [9]. Comparés aux mesures accélérométriques de Chida, les résultats théoriques obtenus par éléments finis sont concordants pour de faibles valeurs de $\mathrm{m}_{\mathrm{n}}$ mais présentent de nettes divergences pour les accélérations élevées $\left(m_{h}=0,40\right)$.

\section{3}

\section{État actuel des connaissances en calcul et dimensionnement à la rupture des sols renforcés}

Les méthodes de calcul et de dimensionnement des ouvrages en sols renforcés consistent à vérifier simultanément :

- la stabilité locale ou «interne »;

- la stabilité globale ou d'ensemble.

\section{1}

\section{Stabilité interne}

On rappelle qu'elle consiste à vérifier sous sollicitations statiques, les deux critères de :

- rupture par cassure d'armature, soit :

$$
T_{\max }<R_{n}
$$

- rupture par défaut d'adhérence entre le sol et l'inclusion, soit :

$$
\mathrm{T}_{\max }<\mathrm{R}_{g}
$$

\section{2}

\section{Stabilité globale}

Les calculs se font généralement de manière automatique (par des logiciels du type TARUPT, TALREN, STAR, PROSPER, etc.) pour un modèle géométrique et mécanique donné. La prise en compte de l'action sismique est introduite par analyse pseudo-statique.

Dans cette présentation, on manifeste un intérêt particulier au calcul et au dimensionnement des ouvrages en terre armée soumis à des actions dynamiques.

Dans ce cadre, on rappelle les quatre méthodes actuelles de dimensionnement:

\section{$3,2.1$}

\section{Méthode de Monobe et Okabe}

Cette méthode combine les actions statiques et dynamiques quant à la détermination de la résultante de pression P derrière le massif renforcé. Celle-ci, considérée dans les deux cas (statique et dynamique) comme croissante avec la profondeur z, est donné par:

$$
\mathrm{P}=\frac{1}{2} \gamma \mathrm{H}^{2} \mathrm{~K}_{\mathrm{AE}}
$$

$K_{A E}$ est un coefficient qui associe les actions statiques et dynamiques et qui dépend de l'accélération.

\section{9 .9}

\section{Méthode de dimensionnement de Richardson}

Richardson ajoute à l'effort statique $\mathrm{P}_{\mathrm{s}}$ une action dynamique $\mathrm{P}_{d}$ de la forme :

$$
P_{d}=\frac{9}{8} K_{0} \cdot \gamma H^{2} \cdot m_{h}
$$

\subsection{3}

\section{Méthode pseudo-statique de dimensionnement}

La méthode pseudo-statique associe aux efforts statiques une force d'inertie $\mathrm{F}$ définie à partir d'une courbe de rupture probable et délimitant la zone instable sous chargement statique. Dans l'hypothèse de la spirale logarithmique $(\mathrm{D}=0,3 \mathrm{H}$, pour les ouvrages en Terre Armée), la force d'inertie F est proportionnelle au poids $W$ de la zone en mouvement, et on a :

$$
F_{1}=W \cdot m_{h}=\frac{9}{40} \gamma H^{2} \cdot m_{h}
$$

\subsection{4 \\ Méthode de Seed et Mitchell}

Les auteurs font une synthèse des deux dernières méthodes et introduisent deux types d'action dynamique (Fig. 7) :

- une force d'inertie $F$, uniformément répartie sur toute la hauteur $\mathrm{H}$ du massif et calculée pour une largeur de la zone instable de H/2. On obtient (Fig. 7 a) :

$$
F_{i}=\gamma D^{\prime} \cdot H \cdot m_{h}=\frac{1}{2} \gamma \cdot H^{2} m_{h}
$$

- une force résultant de la poussée latérale du remblai derrière le mur armé et analogue à celle donnée par Monobe et Okabe, soit (Fig. 7 b) :

$$
\mathrm{P}_{\mathrm{d}}=\frac{3}{4} \mathrm{~m}_{\mathrm{h}} \gamma \cdot \mathrm{H}^{2}
$$



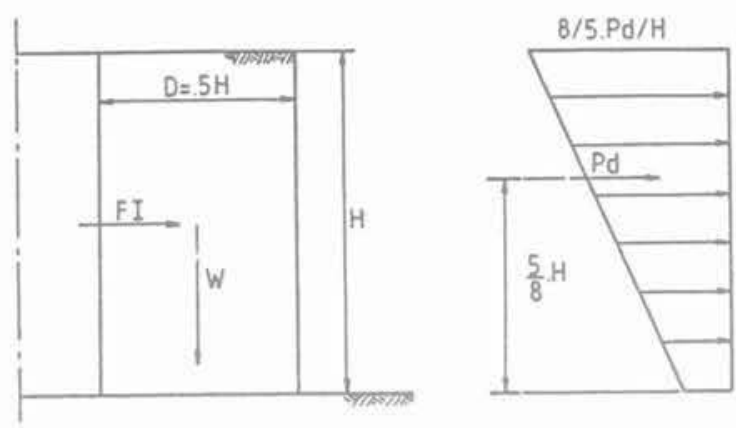
a. Force d'inertie
b. Poussée du remblai
a. Inertia force
b. Lateral pressure

\section{4}

\section{Réflexions sur les modifications des méthodes actuelles de dimensionnement dynamique}

\section{1}

\section{Analyse des différentes méthodes actuelles}

L'analyse des différentes méthodes que nous venons d'évoquer conduit aux remarques suivantes : - la méthode de Monobe et Okabe n'explicite pas la poussée latérale d'origine dynamique. Elle ignore, comme la méthode de Richardson, la force d'inertie; - la méthode de calcul pseudo-statique a l'inconvénient de supposer que la distribution des tractions dans les armatures n'est pas qualitativement modifiée par l'action dynamique. Ce qui n'est compatible ni avec les mesures expérimentales sur modèles réduits tridimentionnels de Richardson [9] ni avec les résultats numériques, Dhouib et al. [7]. La largeur de la zone instable est, en effet, augmentée par l'action dynamique. Cette méthode omet également la poussée horizontale du remblai derrière le massif armé;

- lapproche de dimensionnement proposée par Seed et Mitchell a l'avantage d'introduire les deux forces d'inertie et de poussée, Cependant, elle surestime la largeur de la zone potentiellement instable $\left(D^{\prime}=H / 2\right)$ pour le calcul de la force d'inertie. L'examen des évolutions des déplacements et du phénomène d'amplification (en particulier en partie supérieure du massif), montre que cette méthode est la plus compatible avec la distribution (qualitative) de la poussée horizontale dynamique.

\section{2}

\section{Réflexions sur le dimensionnement des ouvrages en Terre Armée}

Dans cette optique, on envisage d'étudier les dimensionnements vis-à-vis de la stabilité «interne » et de la stabilité globale.

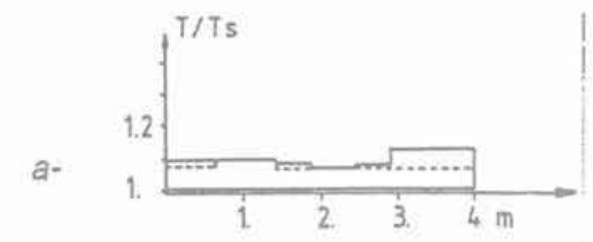

b.

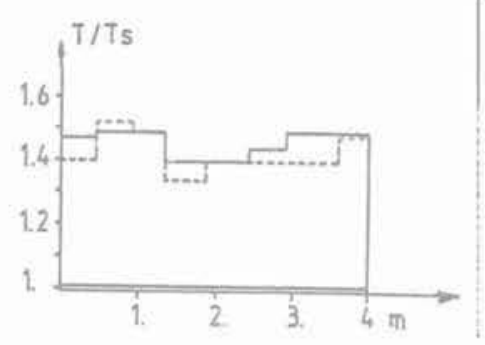

a. $m_{h}=0,12$

b. $m_{n}=0,46$

FIG, 8

Évolution de la traction totale $T$ (par la valeur statique $T$ ) dans I'armature $\mathrm{n}^{\circ} 7$ Evolution of the whole tensile force $\mathrm{T} /$ the static value $T_{\text {s }}$ in the strips $n^{\circ} 7$

Sur la figure 8, on représente l'évolution du rapport $T / T$, en fonction de $m_{n}(=0,12$ et 0,46$)$. On remarque que l'incrément de la traction résultant de l'action dynamique $\Delta \mathrm{Td}$ est, en moyenne, proportionnel à son homologue statique $T$. Ce résultat a été déjà obtenu par Richardson dans les essais sur modèles réduits tridimentionnels et on peut retenir :

$$
\Delta \mathrm{Td}=\mathrm{m}_{\mathrm{h}} \cdot \mathrm{T}_{\mathrm{s}}
$$

$\mathrm{m}_{\mathrm{h}}$ étant la valeur maximale du rapport $\mathrm{a}_{\mathrm{h}} / \mathrm{g}$ induite dans le mur.

\section{Dimensionnement « interne}

Le critère de résistance à la rupture par cassure d'armature s'écrit alors :

$$
\mathrm{T}_{\max }=\left(1+\mathrm{m}_{\mathrm{n}}\right) \mathrm{T}_{\mathrm{s}}<\mathrm{R}_{\mathrm{n}}
$$

Le critère de résistance au glissement sol - armature s'exprime, de manière découplé, à savoir :

$$
\begin{gathered}
\mathrm{T}_{\max }<\mathrm{R}_{\mathrm{g}} \text { (statique) } \\
\Delta \mathrm{T}_{d}<\mathrm{R}_{\mathrm{v}}^{\prime} \text { (dynamique) }
\end{gathered}
$$

avec :

$\mathrm{R}_{\mathrm{g}}^{\prime}<\mathrm{R}_{\mathrm{g}}$ (car les effets d'ondes conduisent à une diminution des contraintes verticales instantanées et, en conséquence, du coefficient de frottement apparent dynamique).

\section{Dimensionnement global}

Les différents mécanismes de rupture (par cassure d'armature ou par glissement sol - inclusion), observés sur modèles réduits tridimensionnels, Richardson [9] montrent que des plans de cisaillement se développent en pied du massif armé.

Ces observations et les calculs théoriques effectués, permettent de considérer, dans le cas de massifs renforcés par des inclusions quasi inextensibles, l'hypothèse de la spirale logarithmique tout en mentionnant 
que la zone instable s'élargit et s'approfondit avec l'action dynamique.

Cet élargissement peut être pris égal à $m_{n} \cdot \mathrm{H} / 2$ (Dhouib et al. [5 à 7]) valeur suggérée également par des expérimentations japonaises. Ce qui conduit à :

$$
\mathrm{D}^{\prime}=\mathrm{D}+\mathrm{m}_{\mathrm{b}} \cdot \mathrm{H} / 2
$$

Quant à l'approfondissement $\mathrm{h}$, on retient (Dhouib et al. [5 à 7]):

$$
\mathrm{h}=(1+\mathrm{k}) \cdot \mathrm{H} / 2
$$

(au lieu de H/2 en partie supérieure du mur)

Le poids $W$ de la zone instable est alors:

$$
W=\gamma \frac{D^{\prime} H}{4}(k+3)
$$

On remarque bien que, lorsque :

$$
\begin{aligned}
k & =0 \\
D^{\prime} & =D
\end{aligned}
$$

on a :

$$
\mathrm{W}=\frac{3}{4} \gamma \mathrm{DH} \text { (statique) }
$$

La force d'inertie $F$, est, pour des faibles valeurs de $m_{h}\left(m_{h}^{3} \rightarrow 0\right)$, donnée par:

$$
\mathrm{F}_{\mathrm{i}}=\frac{2}{9} \gamma \mathrm{H}^{2} \mathrm{~m}_{\mathrm{h}}\left(2 \mathrm{~m}_{\mathrm{h}}+1\right)
$$

Il convient de noter, que pour :

$$
\begin{aligned}
& K=0 \\
& D^{\prime}=0,3 H+m_{h} H / 2=\frac{H}{10}\left(3+5 m_{h}\right)
\end{aligned}
$$

on obtient (Bastick et al. [1]) :

$$
F_{1}=\frac{9}{40} \gamma H^{2} \frac{m_{h}}{3}\left(3+5 m_{h}\right)
$$

On envisage d'établir dans ce qui suit, une symthèse comparative entre la méthode de dimensionnement pseudo-statique et la méthode de Seed et Mitchell et les modifications que nous proposons d'apporter à la méthode pseudo-statique.

Pour ce faire, on représente sur la figure 9, la distri-

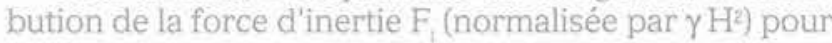
différentes valeurs de $m_{n}$. On constate que les valeurs des forces d'inertie F calculées par la méthode des éléments finis et celles des modifications que nous avons apportés à la méthode pseudo-statique (Fig. 9 a) sont bien encadrées par les valeurs obtenues par cette dernière et la méthode de Seed et Mitchell (Fig. 9 b).

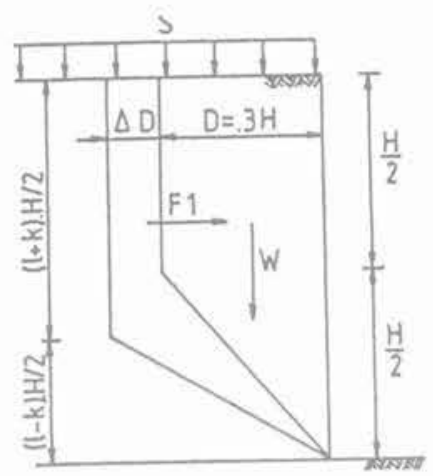

Méthode de Seed et Mitchell Méthode pseudo-statique

a. Calcul de la force d'inertie.

a. Predicted inertia force.

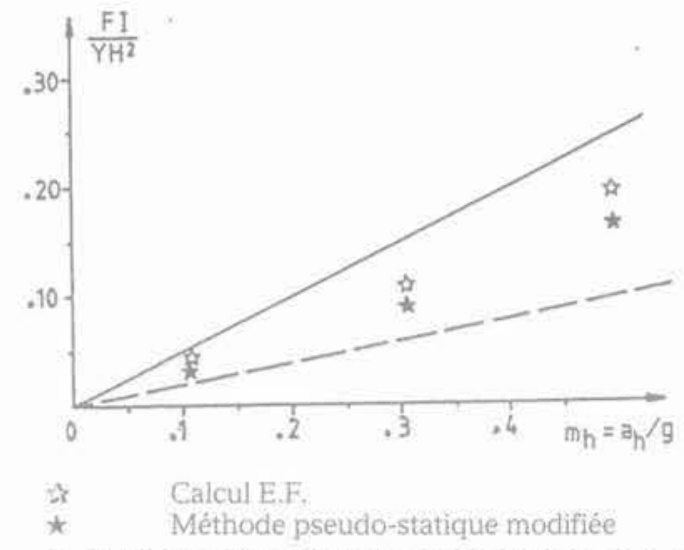

b. Distribution de la force d'inertie en fonction de $m_{h}$. b. Evolution of the inertia force.

FIG.9 Évolution de la force d'inertie : étude comparative. Comparative evolution of inertia force.

La distribution de la poussée latérale dynamique $\mathrm{P}_{\text {}}$ (normalisée par $\gamma \mathrm{H}^{2}$ ) en fonction de $\mathrm{m}_{n}$ donnée sur la figure 10 conduit à noter les points suivants :

- la poussée latérale dynamique $\mathrm{P}_{\mathrm{d}}$ est maximale à une distance de $0,5 \mathrm{H}$ derrière le parement et non pas à $\mathrm{D}+\mathrm{m}_{h} \mathrm{H} / 2$. Ceci justifie la distribution proposée par Seed et Mitchell.

Par contre, l'évolution donnée par Richardson concorde mieux avec une largeur de la zone instable de $\mathrm{D}+\mathrm{m}_{\mathrm{i}} \cdot \mathrm{H} / 2$;

- comparées aux résultats obtenus par la méthode de Seed et Mitchell, les valeurs de la poussée latérale dynamique sont sous-estimées par l'approche linéaire équivalente. Cette diminution s'accentue avec les valeurs croissantes de $m_{n}$.

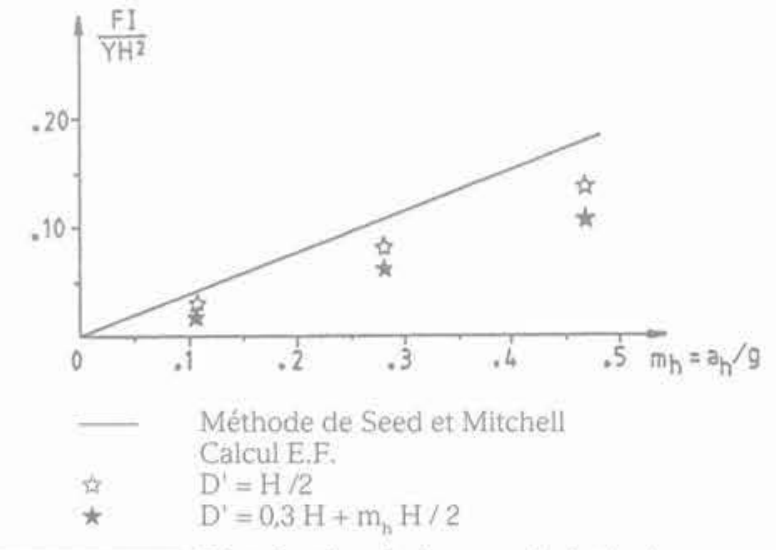

FIG, 10 Distribution de la poussée latérale dynamique en fonction de $\mathrm{m}_{\mathrm{b}}$ : étude comparative.

Dynamic lateral pressure evolution : comparative study. 


\section{Synthèse - conclusion}

Il a été constaté à travers cette présentation, que l'action dynamique a, pour effet, d'une façon générale : - d'augmenter la traction dans l'inclusion;

- de modifier la distribution des tractions maximales en fonction de la profondeur:

- d'élargir la zone instable;

- d'accentuer les déplacements en tête du massif armé et de faire apparaitre un phénomène d'amplification en partie supérieure du mur.

Le calage des résultats théoriques des calculs par éléments finis sur les mesures expérimentales de Chida montre que l'analyse linéaire équivalente proposée est d'autant plus fiable que les effets dynamiques sont faibles,

En effet, la comparaison des résultats du phénomène d'amplification avec les mesures expérimentales de Chida prouve qu'une telle approche conduit à des valeurs concordantes pour un niveau d'accélération faible $\left(m_{h}=a h / g=0,10\right.$ à 0,15$)$.

Une étude plus fine de l'effet de certains paramètres (extensibilité des armatures, rigidité de la paroi...) sur les tractions dynamiques est en cours d'élaboration. En effet, nous avons constaté que les tractions dynamiques calculées par éléments finis diminuent avec l'extensibilité des armatures. Par contre, elles augmentent avec la rigidité de la paroi; ceci s'explique, a priori, par l'augmentation, comme sous sollicitations statiques, de la poussée latérale au voisinage de la paroi rigide.

Une telle étude nécessite, afin d'être validée, des expérimentations en vraie grandeur qui sont, en dehors des essais de Chida, rarement réalisées à ce jour. L'usage des mesures sur modèles réduits tridimensionnels reste, malgré l'intérêt qualitatif qu'il représente, limité par les problèmes de similitude.

\section{Bibliographie}

[1] Bastick M. Schlosser F. - Comportement et dimensionnement des ouvrages en terre armée. Comptes rendus du premier colloque national de Génie Parasis. mique, Paris, 1986.

[2] Bastick M. - Résistance aux séismes des ouvrages en Terre Armée, Revue Francaise de Géotechnique, 1986.

[3] Chida S., Minami K, - Reinforced Earth Technique: its Outline and Laboratory Test, Publication interne du ministère des Travaux publics japonais. August 1980.

[4] Chida S., Minami K. - Tests with regard to the stability of the fill constructed by the reinforced earth technique, Publica- tion interne du ministère des Travaux publics japonais, (1982).

[5] Dhouib A., Robinet J.-C. - Domains of Behaviour and Behaviour of reinforced soils, International Conference Series on Advances in Numerial Methods in Engi. neering, Theory and Applications, Numeta, 1987

[6] Dhouib A. Robinet, J.-C. - Comportement des sols renforcés en chargements monotones et répétitifs, Applications à la Terre Armée, Congrès franco-polonais de Mécaniques des sols, Grenoble, 1987.

[7] Dhouib A. Knochenmus G. - Behaviour study and design of reinforced earth structures under dynamic efforts, Second International Conference on
Recent Advances in Geotechnical Earthquake engineering and soil dynamics, St-Louis, Missouri, USA, 1991.

[8] Pecker A. - Dynamique des sols, Paris, Presses de l'ENPC, 1984.

[9] Richardson G.N., Feger D., Fong A. Associate members ASCE, Lee K.L. Seismic Testing of Reindroced Earth wall : ASCE, January, 1977

[10] Schlosser F., Nguyen Than Long Comportement de la Terre Armée dans les ouvrages de soutènement. Congrès de Mécanique des sols, Moscou, 1973.

[11] Schlosser F., Dormieux L. - Talus et soutènement en dynamique des sols. Revue Française de Géotechnique, 1986. 